\title{
DEVELOPMENT OF A STAND ALONE MONITORING SYSTEM (S.A.MO.S.)
}

\author{
C. Kosmatopoulos, N. Tsagourias
}

Electronics Lab., Physics Department, Aristotle University of Thessaloniki

54006 Thessaloniki, Greece, kosmatopoulos@physics.auth.gr

Abstract: Environmental monitoring is nowadays a common instrumentation application, not only in cases where scientific information is needed, but also for pollution control and development planning of certain sensitive areas. This paper describes the development of a Stand Alone Monitoring System (S.A.MO.S.) which is actually a complete monitoring station for environmental measurements in rivers, lakes, lagoons and other sensitive eco-systems. S.A.MO.S. is capable of performing scheduled measurements by sensors or other independent electronic measuring instruments, and stores data locally in a memory module. Measurement data may then be transferred to a PC computing system for further analysis either by using cellular phone network or via a UHF transceiver. Validation of measurements is achieved by system's self check control and warning signals can be sent to authorized personnel in case of errors. The basic features and the configuration of this system are presented in this work.

Keywords: - autonomous monitoring system, data logger, remote environmental monitoring, observation of remote sites

\section{INTRODUCTION}

Over the past several decades, the collected experience of scientists has led to the awareness that ecosystems are sensitive to a whole set of interactive parameters [1]. Monitoring is critical for environmental management, and developing the environmental strategy as well as capturing current status of ecosystem is important since rapid changes in environmental conditions may be detected $[2,3]$.

S.A.MO.S. is a fully self-efficient environmental monitoring station, capable of supporting practically almost all types of sensors [4] for measurements of water parameters (conductivity, $\mathrm{pH}$, dissolved oxygen, flow, etc) and others as well (meteorological, geological, hydrological, etc). S.A.MO.S. is also equipped with digital output control ports, and a timing input channel ensuring optimized control and function of these sensors. The system stores the acquired information over an extended time period until it is collected and processed. Remote data and command transferring can be achieved by a conventional or mobile phone connection. Authorized personnel may then get connected to the station and perform all kinds of actions: collect remotely all data, via phone or Internet, reprogram measurement sequences, and also perform an overall control to the system's operating function. A laptop connection output enables service/maintenance, or data transfer on the spot. This system is actually the new version of instrumentation systems development of the same re- search team [5-7], which are currently being used in different places in Greece [8].

S.A.MO.S. is based on an R.T.O.S. (Real Time Operating System) which is developed for an 8-bit microcontroller and is capable of performing scheduled measurements and storing them with a time and date stamp, in a removable non-volatile memory.

Intelligent techniques of data processing may also be applied, while focus is given on methods of measurements validation by checking for improper values or "strange" sequences compared to the expected measurements.

S.A.MO.S. is also capable of performing a selfcheck control on scheduled time periods and store the results along with actual measurements. Warning signals can be transmitted to the authorized scientists in case of errors.

A supervisory system by means of a special software applied, can automatically collect and store data from stations permanently, analyzing and publishing them to the Internet, even assisting with system's preventive maintenance. Authorized personnel may also call the supervisory system when an alarm situation occurs for a detail setup.

The system has been designed in a modular way which makes possible any further hardware expansion and software re-usability.

\section{HARDWARE DESIGN}

A functional diagram of S.A.MO.S. is shown in Fig. 1. It is a useful reference that shows the 


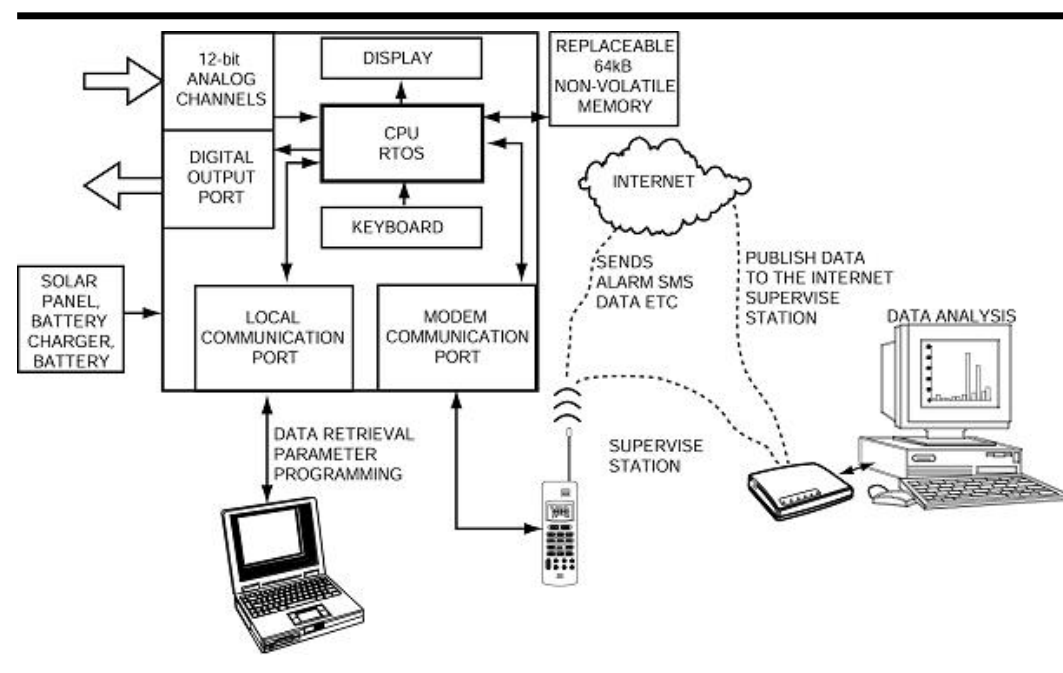

Fig. 1 - System's block diagram
S.A.MO.S. has a 16-keys keyboard which allows on site programming system's parameters (i.e. time and date etc) and measurement sequences. Using a keyboard we can perform immediate measurements to assure on-site measurements' accuracy. An alphanumeric display facilitates viewing real-time and historical data, as well as keyboard entries (i.e. time of day and other system parameters).

System's stand-alone operation is achieved by a power source block which consists of a data path in the module and explains the function of many of the system's components.

A weatherproof enclosure protects the system from humidity and extremely harsh weather conditions.

The system is capable of supporting practically almost all types of sensors such as:

- Pressure transducers

- Shaft encoders

- Ultrasonic level sensors

- Flow meters

- Conductivity sensors

- $\mathrm{pH}$ sensors

- Thermistors

- Tipping bucket and weighing rain gages

- Wind vanes

- Anemometers

- Relative humidity sensors

- Pyranometers

- Leaf wetness sensors

- Fuel moisture/temperature sensors

The first step is to acquire the sensor signal and convert it to digital data [9]. In Figure 1, all the signal conditioning circuitry has been lumped into one block which is referred as the analog channels block. Analog channels can be configured to provide singleended or differential measurements using 12-bit resolution A/D converters. Autozero and autocalibration is performed during system's self check control to ensure the proper $\mathrm{A} / \mathrm{D}$ converters' functionality. The state of sensors is controlled by the datalogger relay-driven digital control output port. A timing input channel is used in conjunction with those sensors which produce output in time-domain.

A digital input port is used to sense the status of external devices or to capture discrete digital signals. Digital signals can be configured to act as pulse counters (such as rain meters) or interrupt inputs (warning signals). solar panel, a battery charger/regulator and a $12 \mathrm{~V}$ battery. The battery voltage is periodically measured, recorded, and transmitted by the datalogger for self-monitoring purposes (proper system's operation).

Replaceable EEPROM memory is used to record data measured from channels. The storing capability of memory is 4000 measurements for each channel (analog, time, discrete digital). EEPROM memory has several internal pointers which are used to keep track of data for both storage and retrieval operation. Replaceable memory also contains the programmable parameters for the channels such as measurement rate, measurement quantity etc, which can be carried from the site of experiment to the laboratory and plugged in the reading card to perform extended data processing.

The core of the system is a micro-controller unit which is actually controlling the whole system's operation [10]. The micro-controller is principally composed of a central processing unit (CPU), I/O circuits, timer, serial interfaces and internal random access memory. The microcontroller is driven by a low frequency crystal oscillator, which in relation to a "smart" firmware offers a considerable reduction to power consumption, a feature that is essential to battery-powered applications.

S.A.MO.S is able to communicate with local or remote computers, and personnel in two ways:

1. A local communication port is used for local data collection, local system setup, status report, and diagnostics check.

2. A modem communication port is used for remote programming and interactive communication ability. Using modem communication port authorized personnel may access the system at any time to request data collection, status report or diagnostics check. When "alert" conditions occur, system automatically dials up to preset phone numbers to 
notify key personnel. S.A.MO.S. dials numbers in sequence until it receives verification that an authorized person has been reached. Users can then initiate emergency procedures if necessary. In modem communication port a cellular phone is connected which is used from system for periodically data transfer to a remote computer for further analysis.

\section{SOFTWARE DEVELOPMENT}

Software is developed to meet the different needs of the system's requirements. System's software is divided in three different parts:

- System's firmware, which is installed in microcontroller ROM and it's responsible with micro-controller hardware for proper system operation.

- Communication software, which is capable to performall communication tasks including local and remote communications.

- Data analysis and Internet publication software which enables data collection, presentation as well as generation of reports which facilitates displaying information on Internet pages.

System's firmware is based on an R.T.O.S. (Real Time Operating System)-which is developed for an 8-bit micro-controller- offers very fast software development and gives flexibility that allows new modules to be added without interfering with those already in place whenever a system's firmware upgrade occurs. A software implementation of real-time clock assures accurate timekeeping. The operating system executes defined tasks at specific, regular time intervals. These tasks are written so that they run immediately and do not require code to determine the timing of their execution. Rather, firmware developer already schedules the rate of execution. This operating system is ideal for many predicted duration routines with few or short duration interrupts. Firmware is also capable of performing scheduled measurements and store them with a time and date stamp, in a removable non-volatile memory. Intelligent techniques of data processing are also included, while focus is given on methods of measurements validation by checking for improper values or "strange" sequences compared to the expected measurements. For serial communication ports firmware includes error detection and correction algorithms to provide confidence that critical data is received/transmitted correctly. The firmware supports "sleep mode" which reduces the power consumption. The system's "sleep mode" is only interrupted when measurements are to be taken or to interrogate the communication network for any requests.

Communication between the monitoring system and the receiving computer either local or remote is accomplished by means of a special PC-compatible communication software. This software is responsible for data retrieval and system's configuration using a user-friendly interface. Remote communication software is also responsible for autoanswer to any warning signals coming from the site of experiment/meas urement.

Software for data analysis support from one hand algebraic, statistical and transcendental functions allowing extended processing of data needed to develop policies based on current scientific understanding of ecosystem processes.

The final element of the environmental monitoring software chain is based on a continuously updated database. This database contains data retrieved from one or more remote sites. The specially developed software is used for two purposes: for mathematical analysis applying functions such as algebraic, statistical, and transcendental of collected data and also for data extraction which can be used either with third-party analysis software tools or for internet publication. The Internet publication is based on an automatic generation of HTML pages and transferring them to a web server which users will be able to access interactively via the Internet.

\section{CONCLUSION}

The operating characteristics and the electronic configuration of a Stand Alone Monitoring System (S.A.MO.S.) is presented in this work. This type of automated monitoring instruments can be used as remote stations, which may continuous ly monitor the value of various parameters of the water in environmentally sensitive ecosystems (lagoons, rivers, lakes). The prototype of this electronic system has been developed at the Electronics Lab., Aristotle University of Thessaloniki, and is currently in the test phase to be placed in certain remote areas. Each S.A.MO.S unit is actually a remote, computerized, field-monitoring station, which may measure, store and then transfer wirelessly the results of all measurements to a base station for storage, analysis and evaluation. Each of these stations is selfefficient in terms of power and communications, and capable of measuring practically any parameter that can be measured electronically (different, independent instruments may connected to this type of stations). The basic technical characteristics of S.A.MO.S. can be summarized as following:

1. High accuracy measurements, time and date stamped derived from eight channels of measurements. 
2. Sensor's control achieved by all of the digital output control ports.

3. Storing capability to up to 4000 measurements in a replaceable non-volatile memory module.

4. Compatibility with practically all types of electronic sensors.

5. Upgradeable firmware that can be downloaded from a remote site.

6. Full access to the system via a cellular or UHF communication channel.

\section{REFERENCES}

[1] Environment Division Office of Science and Technology Policy Executive Office of the President of the USA, "Integrating the nation's environmental monitoring and research networks and programs: A proposed framework", March 1997.

[2] "The environmental monitoring of large international companies. How and what is monitored and why", Atie H. Verschhor, Lucas Reijnders, Journal of Cleaner Production 9 (2001) 43-55.

[3] Rose KA, Smidt EP. "Experimental Design: the neglected aspect of environmental monitoring". Environ Manage 1992; 16(6):691-700.

[4] Randy F. "Understanding smart sensors". Boston (MA): Artech House, 1994.

[5] Zachos N., Kosmatopoulos C. and Laopoulos Th., A wireless network of remote mea- suring stations: Application in water level monitoring, IEEE International Symposium on Industrial Electronics, ISIE'95, Athens, Greece, 1995

[6] Kosmatopoulos C., Papageorgiou Ch., Tsagourias N. and Laopoulos Th. Development of a remote environmental monitoring system (R.E.MO.S.), IMEKO TC-4 International Symposium on the Developments in Digital Measuring Instrumentation, Naples, Italy, Sept. 1998

[7] Laopoulos Th., Kosmatopoulos C. and Albanakis $K$., The remote monitoring system of the Santorini volcano: from sea-level monitoring to an Integrated Remote Volcanological Monitoring Network (IRVMN), 2nd Workshop on European Laboratory Volcanoes, Santorini, Greece, 1996

[8] Albanakis K., Laopoulos Th. and Kosmatopoulos C., Sea level monitoring network of Santorini volcanic islands, 2nd Workshop on European Laboratory Volcanoes, Santorini, Greece, 1996

[9] "Miniature data loggers for remote measurement of body temparature in medium-sized rodents", Peter R. Kamerman, Luca C. Di Zio, Andrea Fuller, Journal of Thermal Biology, 26 (2001) 159-163.

[10] "Monitoring a greenhouse using a microcontroller-based meteorogical data-acquisition system", S. Ameur, M. Laghrouche, A. Adane, Renewable Energy 24 (2001) 19-30

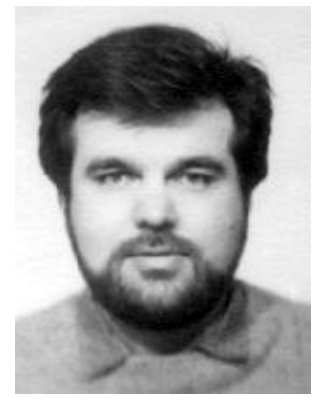

C.A. Kosmatopoulos received the BSc degree in physics and the PhD degree in electronics both from the Aristotle University of Thessaloniki, Greece, in 1981 and 1991, respectively.

He has been a member of the academic staff at the Electronics Laboratory of the

Physics Department, Aristotle University of Thessaloniki, Greece since 1981. He works as a lecturer for the Electronic Laboratory of the Physics Department since 1991. His main research interests lie in the field of electronic circuits and power electronics.

Moreover since 1993 he has been a member of a research team working on instrumentation systems development especially for measuring environmental parameters.

He has published a number of papers in international journals and magazines and conference proceedings. He has edited two books and has been the supervisor to a number of graduate and postgraduate students' thesis.

He is married and he is the father of two children.

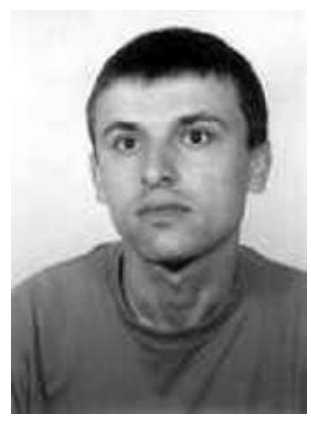

Nick Tsagourias graduated in Electronic Engineering degree by the Technological Educational Institute, ThessaIoniki, in 1997 and obtained the MSc degree in New Electronics Technologies from University of loannina in 2001.

Since 1993 , he has been in collaboration with the Electronic Lab of Physics Department of Aristotle University, where he works on telemetering systems and networks. Since 1998 he has been working as an educator in Technological Educational Institute where he organizes and teaches two courses called 'Microcomputers' and 'Microcomputers Applications'

His currentresearch interests include programmable circuits and systems, telemetering systems and methods and networks. He has co-authored on three conference papers. 